Preprint DFPD 01/TH/17

hep-th/0105102

\title{
Introduction to the Superembedding Description of Superbranes
}

\author{
Dmitri Sorokin \\ INFN, Sezione di Padova, via F. Marzolo 8, 35131, Padova, Italia \\ and Institute for Theoretical Physics, National Science Center, KIPT, Kharkov, Ukraine
}

\begin{abstract}
Basics of the geometrical formulation of the dynamics of supersymmetric objects are considered and its relation to conventional formulations of superbranes is discussed. In particular, we demonstrate how the kappa-symmetry of the Green-Schwarz formulation shows up from local worldvolume supersymmetry, and briefly discuss applications of the superembedding approach.
\end{abstract}

\section{INTRODUCTION}

Superembedding is an elegant and geometrically profound approach which is based on a supersymmetric extension of the classical surface theory to the description of superbrane dynamics by means of embedding worldvolume supersurfaces into target superspaces (see [1] for a detailed review).

The superembedding approach arose as a proposal to solve the problem of the covariant quantization of the Green-Schwarz superstring by combining in a more general formulation main properties of both the Neveu-Schwarz-Ramond (NSR) and the Green-Schwarz (GS) formulation, and by now it has developed into a generic geometrical method for formulating the theory of superbranes.

Being manifestly doubly supersymmetric (on the worldvolume and in target superspace) the superembedding approach has explained the origin and the nature of the fermionic $\kappa$-symmetry of the GS formulation as a manifestation of the conventional local supersymmetry of the worldvolume, and thus solved the problem of infinite reducibility of the $\kappa$-symmetry by realizing it as an irreducible extended worldvolume supersymmetry [2]. This stimulated progress in the covariant quantization of the GreenSchwarz superstring mainly due to persistent work of N. Berkovits [3].

The superembedding approach established or clarified a classical relationship between various formulations of the dynamics of superparticles and superstrings, such as the NSR and the GS formulation [4], the twistor [5] and harmonic [6] descriptions.

This approach has proved to be a universal and powerful method applicable to the description of all known supersymmetric branes, in particular, to those of them for which standard methods encountered problems because of their specific structure, such as the 5-brane of M-theory. The superembedding methods allowed, for the first time, to derive the complete set of covariant equations of motion of the M5-brane [7]. And only later 
these equations were obtained [8] from the M5-brane action [9] based on a different technique adapted to deal with self-dual fields.

The superembedding formulation has also proved to be useful for studying the "brany" mechanism of partial supersymmetry breaking [10] by giving a geometrical recipe $[11,12,13]$ for constructing covariant worldvolume supersymmetric actions for superbranes. Upon gauge fixing worldvolume superdiffeomorphisms these actions become those of effective field theories with non-linearly realized spontaneously broken supersymmetries. This has demonstrated an intrinsic link of the superembedding approach and the method of nonlinear realizations developed in application to supersymmetric theories in [14] (see [15] for a review and references).

In this contribution I would like to make an introduction into the superembedding formalism and describe its basic properties.

\section{DOUBLE SUPERSYMMETRY}

As it has been mentioned in the Introduction the superembedding description of superbrane dynamics is formulated in such a way that it possesses manifest supersymmetry on the brane worldvolume and in target superspace, and thus has properties of both the NSR and the GS formulation.

\section{The Neveu-Schwarz-Ramond formulation}

In the NSR formulation, which describes spinning particles [16] and spinning strings $[17]^{1}$, the worldline or worldsheet of the spinning object is a supersurface $\mathcal{M}_{w}$ parametrized by bosonic coordinates $\xi$ and fermionic coordinates $\eta$ which we will collectively call $z^{M}=(\xi, \eta)$. Depending on the model considered $\mathcal{M}_{w}$ may have a various number of fermionic directions $\eta$. For simplicity we here take only one $\eta$. The dynamics of the spinning object is described by embedding $\mathcal{M}_{w}$ into a bosonic target space-time $M_{T}$ parametrized by coordinates $X^{\underline{m}}(\underline{m}=0,1, \cdots, D-1)$. In the classical problems the number of space-time dimensions can be arbitrary, but for quantum consistency the spinning string, whose worldsheet has one or two fermionic directions, must live in a ten-dimensional target space.

The motion of the spinning object is described by the image of $\mathcal{M}_{w}$ in $M_{T}$

$$
X^{\underline{m}}\left(z^{M}\right)=x^{\underline{m}}(\xi)+i \eta \chi^{\underline{m}}(\xi),
$$

where $x^{\underline{m}}(\xi)$ is associated with the bosonic degrees of freedom of the spinning particle or string in $M_{T}$, and the Grassmann-odd vector $\chi^{\underline{m}}(\xi)$ is associated with its spin degrees of freedom.

\footnotetext{
${ }^{1}$ No spinning branes have been consistently constructed so far [18].
} 
The NSR formulation is invariant under worldsheet superdiffeomorphisms $z^{M} \rightarrow z^{M}\left(z^{M}\right)$, which include $\mathcal{M}_{w}$ bosonic reparametrizations

$$
\delta \xi=a(\xi)
$$

and local worldsheet supersymmetry

$$
\delta \eta=\kappa(\xi), \quad \delta \xi=i \kappa(\xi) \eta .
$$

The presence of the local symmetries (2) and (3) implies that the dynamics of the spinning objects is subject to bosonic and fermionic first-class constraints, respectively, which I will schematically write down in the form

$$
\left(\partial x^{\underline{m}}\right)^{2}=0, \quad \partial x^{\underline{m}} \chi_{\underline{m}}=0 .
$$

The bosonic constraint in (4) is the mass shell or the Virasoro conditions, and the fermionic constraint produces, upon quantization, the Dirac equation for the spin wave functions of the dynamical system.

The NSR formulation does not have target-space supersymmetry. The latter appears, in the case of the spinning string, only at the quantum level upon imposing the GliozziScherk-Olive projection. The merit of this formulation is that it is covariantly quantizable.

\section{The Green-Schwarz formulation}

This formulation is applicable to all known superparticles, superstrings and superbranes $^{2}$.

Now the worldvolume $\mathcal{M}_{w}$ is a $(\mathrm{p}+1)$-dimesnional bosonic surface parametrized by the coordinates $\xi^{m}$ ( $\left.m=0,1, \cdots, p\right)$ and the target space, into which $\mathcal{M}_{w}$ is embedded, is a superspace $M_{T S}$ parametrized by bosonic coordinates $x \underline{m}(\underline{m}=0,1, \cdots, D-1)$ and by an appropriate number of fermionic coordinates $\theta \underline{\alpha}(\underline{\alpha}=1, \cdots, 2 n)$

$$
Z_{T S}^{\underline{M}}(\xi)=\left(x^{\underline{m}}(\xi), \quad \theta^{\underline{\alpha}}(\xi)\right)
$$

The GS formulation is manifestly invariant under bosonic $\mathcal{M}_{w}$ reparametrizations $\xi \rightarrow \xi^{\prime}(\xi)$, and target space superdiffeomorphisms $Z^{\underline{M}} \rightarrow Z^{\prime} \underline{M}\left(Z^{\underline{M}}\right)$, which in the case of flat target superspace reduce to the translations along $x^{\underline{m}}$ and to the global target-space supersymmetry transformations

$$
\delta \theta^{\underline{\alpha}}=\varepsilon^{\underline{\alpha}}, \quad \delta x^{\underline{m}}=i \bar{\theta} \Gamma \underline{\underline{m}} \delta \theta
$$

\footnotetext{
2 The name "Green-Schwarz" used for this formulation should be regarded as cumulative, since for different extended objects it has been developed by different people. This also concerns the "NSR" fromulation. A detailed list of references the reader may find in [1].
} 
There is also another (non-manifest) local worldvolume fermionic symmetry, so-called $\kappa$-symmetry, inherent to the GS formulation. This is an important symmetry which implies and reflects the existence of supersymmetric BPS brane-like solutions of corresponding supergravity theories. It is thus responsible for the 'brane scan' (i.e. which brane lives in which target superspace). The $\kappa$-symmetry was first observed in the case of superparticles [19] and has the following generic form of transformations (in flat target superspace):

$$
\delta \theta^{\underline{\alpha}}=\Pi^{\underline{\alpha}} \underline{\beta}^{\beta}{ }^{\underline{\beta}}(\xi), \quad \delta x^{\underline{m}}=-i \bar{\theta} \Gamma \underline{m} \delta \theta,
$$

where $\Pi^{\underline{\alpha}}{ }_{\underline{\beta}}$ is a projector matrix $\left(\operatorname{det} \Pi^{\underline{\alpha}}{ }_{\underline{\beta}}=0\right)$, whose form depends on the object under consideration.

Because of the presence of the projector in the $\kappa$-transformations they are infinite reducible, so only half of $\kappa^{\underline{\alpha}}$, i.e. $n$ of the $2 n$ Grassmann spinor components, effectively contribute to the variation of the worldvolume fields. This is a cause of the problem of the covariant quantization of the GS formulation.

To solve the infinite reducibility problem of the $\kappa$-symmetry it is natural to try to find its irreducible realization which is covariant in target superspace. A natural assumption is that this should be an extended local worldvolume supersymmetry (3) with the number of independent parameters equal to the number of independent (irreducible) $\kappa$-symmetries [2]. This reasoning brings us to

\section{The doubly-supersymmetric formulation}

In this formulation the dynamics of superbranes is described by embedding a worldvolume supersurface $\mathcal{M}_{s w}$ parametrized by the coordinates $z^{M}=\left(\xi^{m}, \eta^{\alpha}\right)$ $(m=0,1, \cdots, p),(\alpha=1, \cdots, n)$ into a target superspace $M_{T S}$ parametrized by the coordinates $Z^{\underline{M}}=\left(X^{\underline{m}}, \Theta^{\underline{\alpha}}\right)(\underline{m}=0,1, \cdots, D-1),(\underline{\alpha}=1, \ldots, 2 n)$. Note that the number of the Grassmann directions of $\mathcal{M}_{s w}$ is half the number of the Grassmann directions of $M_{T S}$. Such a choice of the supermanifolds for superembedding is caused by our desire to identify $n$ local supersymmetries on $\mathcal{M}_{s w}$ with $n$ independent $\kappa$-symmetries of the GS formulation.

Thus in the doubly supersymmetric formulation the degrees of freedom of the superbranes are described by worldvolume superfields

$$
X^{\underline{m}}\left(z^{M}\right)=x^{\underline{m}}(\xi)+i \eta^{\alpha} \chi^{\underline{m}}(\xi)+\cdots, \quad \Theta^{\underline{\alpha}}\left(z^{M}\right)=\theta^{\underline{\alpha}}(\xi)+\eta^{\alpha} \lambda \underline{\alpha}(\xi)+\cdots,
$$

where $\cdots$ stand for the terms of higher order in $\eta^{\alpha}$. These terms contain auxiliary fields and in addition, for example in the case of the D-branes and the M5-brane, include the gauge fields propagating on the worldvolumes of these branes.

From eq. (8) we see that in the doubly supersymmetric construction the number of degrees of freedom of the superbrane roughly speaking doubles. We now have $x^{\underline{m}}(\xi)$ describing the bosonic oscillations of the brane, the Grassmann 'spin'-vectors $\chi \frac{m}{\alpha}(\xi)$ as in the NSR formulation, the Green-Schwarz fermionic spinor degrees of freedom $\theta^{\underline{\alpha}}(\xi)$ and their bosonic counterparts $\lambda \frac{\alpha}{\alpha}(\xi)$. So if all these worldvolume fields are independent the corresponding models will not describe conventional superbranes. In 
this case one will get, for instance, so called spinning superparticles and spinning superstrings [20] which have more degrees of freedom then the conventional NSR and GS dynamical systems. They also have infinite reducible $\kappa$-symmetry as a fermionic symmetry independent of the local worldvolume supersymmetry.

To reach our goal of interpreting $\kappa$-symmetry as a manifestation of the local worldvolume supersymmetry we should find an appropriate doubly-supersymmetric description of the conventional superbranes. For this we should impose constraints on the superfields (8) which relate their components in such a way that the independent physical degrees of freedom described by these superfields will correspond to the standard GS formulation. The geometrical meaning of these constraints is that they cause the worldvolume supersurface $\mathcal{M}_{s w}$ to be imbedded into the target superspace $M_{T S}$ in a specific way.

\section{THE SUPEREMBEDDING CONDITION}

Before discussing in more detail the geometrical meaning of the superembedding condition let us consider its dynamical consequences in the simplest case of a superparticle propagating in a flat $N=1, D=3$ target superspace. Then the supersurface $\mathcal{M}_{s w}$ of the previous subsection is associated with the superparticle "worldline" having one bosonic (time) and one fermionic coordinate $(\xi, \eta)$, and the target superspace is parametrized by bosonic three-vector coordinates $X \underline{m}(m=0,1,2)$ and Grassmann Majorana twospinor coordinates $\Theta \underline{\alpha}(\underline{\alpha}=1,2)$. The superembedding condition relates the worldline superfields $X^{\underline{m}}\left(z^{M}\right)$ and $\Theta^{\underline{\alpha}}\left(z^{M}\right)$ in the following way

$$
D X^{\underline{m}}-i D \bar{\Theta} \Gamma \underline{m} \Theta=0,
$$

where $D$ is a Grassmann covariant derivative on $\mathcal{M}_{s w}$ which in the case of the superparticles can be chosen to be flat

$$
D=\frac{\partial}{\partial \eta}+i \eta \frac{\partial}{\partial \xi}, \quad\{D, D\}=2 i \partial \xi .
$$

Using the $\eta$-expansion (8) which in the case under consideration does not contain the

"..."-terms we obtain the following relation between the components of the superfields $X^{\underline{m}}\left(z^{M}\right)$ and $\Theta^{\underline{\alpha}}\left(z^{M}\right)$

$$
\begin{gathered}
\partial_{\xi} x^{\underline{m}}-i \partial_{\xi} \bar{\theta} \Gamma^{\underline{m}} \theta=\bar{\lambda} \Gamma^{\underline{m}} \lambda, \\
\chi^{\underline{m}}=\bar{\theta} \Gamma^{\underline{m}} \lambda .
\end{gathered}
$$

From eq. (11) we see that $\lambda \underline{\alpha}(\xi)$ are not independent fields and are expressed in terms of the derivatives of $x^{\underline{m}}$ and $\theta \underline{\underline{\alpha}}$. Moreover in the 1.h.s. of (11) one can recognize the canonical momentum of the superparticle $\Pi^{\underline{m}}=\partial_{\xi} x^{\underline{m}}-i \partial_{\xi} \bar{\theta} \Gamma^{\underline{m}} \theta$ whose square is identically zero

$$
\Pi^{\underline{m}} \Pi_{\underline{m}}=0
$$

because of its so called Cartan-Penrose (or twistor) representation as a bilinear combination of commuting spinor components and $\Gamma$-matrix identities. We conclude that the superparticle is massless. 
In the case of the superstrings the superembedding condition will produce in a similar way the Virasoro constraints, and it will produce the corresponding constraints for the other superbranes.

Eq. (12) implies the relation between the Grassmann vector and the Grassmann spinor variables, so that only one or another can be regarded as describing independent fermionic degrees of freedom. This is a basic relation which allows one to establish a classical correspondence between the NSR and the GS formulation of supersymmetric particles and strings [4].

\section{Local worldvolume supersymmetry versus $\kappa$-symmetry}

Let us now demonstrate how $\kappa$-symmetry appears in the superembedding formulation as a weird realization of the local worldvolume supersymmetry.

The components (7) of the superfields $X^{\underline{m}}\left(z^{M}\right)$ and $\Theta^{\underline{\alpha}}\left(z^{M}\right)$ transform under the local worldline supersymmetry (3) in the standard way

$$
\begin{aligned}
& \delta \theta^{\underline{\alpha}}=-\lambda \underline{\alpha}_{\kappa}(\xi), \quad \delta \lambda^{\underline{\alpha}}=i \partial_{\xi} \theta^{\underline{\alpha}} \kappa(\xi), \\
& \delta x^{\underline{m}}=i \chi^{\underline{m}} \kappa(\xi), \quad \delta \chi^{\underline{m}}=-\partial_{\xi} x^{\underline{m}} \kappa(\xi) .
\end{aligned}
$$

We now substitute into the first equation of (15) the solution (12) of the superembedding condition (9) and observe that, due to the form of the $\theta$-variation (14), the variation of $x^{\underline{m}}$ can be rewritten as follows

$$
\delta x^{\underline{m}}=i \bar{\theta} \Gamma \underline{m} \lambda \kappa(\xi)=-i \bar{\theta} \Gamma^{\underline{m}} \delta \theta .
$$

The next step is to replace the Grassmann scalar parameter of the local supersymmetry by the scalar product of the spinor $\lambda_{\underline{\beta}}$ with a Grassmann spinor parameter $\kappa \underline{\beta}(\xi)$, which is always possible,

$$
\kappa(\xi)=2 \lambda_{\underline{\beta}} \kappa \underline{\underline{\beta}}(\xi),
$$

and to substitute (17) into the $\theta$-variation (14). We thus get

$$
\delta \theta^{\underline{\alpha}}=-2 \lambda \underline{\underline{\alpha}} \lambda_{\underline{\beta}} \kappa^{\underline{\beta}}(\xi) .
$$

We now note that, due to the superembedding condition (11), the bilinear combination of $\lambda$ in (18) is nothing but

$$
\Pi^{\underline{\alpha}} \underline{\beta}=-2 \lambda \underline{\alpha} \lambda_{\underline{\beta}}=\left(\partial_{\xi} x^{\underline{m}}-i \partial_{\xi} \bar{\theta} \Gamma \underline{m} \theta\right)\left(\Gamma_{\underline{m}}\right)^{\underline{\alpha}},
$$

which is the projector matrix in the $\kappa$-symmetry variation of $\theta(7)$. Hence, the local supersymmetry variations (16) and (18) reduce to the $\kappa$-variations (7).

We have thus demonstrated how, in virtue of the superembedding condition (9), the $\kappa-$ symmetry of the GS formulation of the superbranes shows up from the irreducible local worldvolume supersymmetry. 
One might have already noticed the difference in sign in the target-space supersymmetry variations of $x^{\underline{m}}(6)$ and in the worldvolume supersymmetry variations (16) and corresponding $\kappa$-variations (7). The target-space supersymmetry and the local worldvolume supersymmetry (or $\kappa$-symmetry) can be therefore regarded as, respectively, 'left' and 'right' supertranslations of $x \underline{\underline{m}}$.

\section{The geometrical meaning of the superembedding condition}

To understand the superembedding condition from the geometrical point of view let us note that the left hand side of (9) is the Grassmann component of the pull-back onto the superworldline of the target-space vector supervielbein one-form $E^{\underline{a}}=d X^{\underline{a}}-i d \bar{\Theta} \Gamma^{\underline{a}} \Theta$

$$
\left.E^{\underline{a}}\right|_{\mathcal{M}_{s w}}=(d \xi+i \eta d \eta)\left(\partial_{\xi} X^{\underline{m}}-i \partial_{\xi} \bar{\Theta} \Gamma^{\underline{m}} \Theta\right)+d \eta\left(D X^{\underline{m}}-i D \bar{\Theta} \Gamma \stackrel{m}{\Theta}\right),
$$

where $d \xi+i \eta d \eta$ and $d \eta$ form a basis of the supercovariant one-forms (supervielbeins) on the superworldline.

We see that the superembedding condition (9) requires that the pullback of the targetspace vector supervielbein $E^{\underline{a}}$ vanishes along the Grassmann directions of the superworldvolume.

This is the generic requirement for the superembedding to be appropriate to the description of the dynamics of the superbranes.

In general, if we take a supersurface $\mathcal{M}_{s w}$, whose geometry is described by supervielbein one-forms $e^{a}\left(z^{M}\right)(a=0,1, \cdots, p)$ and $e^{\alpha}\left(z^{M}\right)(\alpha=1, \cdots n)$, and a curved target superspace $M_{T S}$, whose geometry is described by supervielbein one-forms $E^{\underline{a}}\left(Z^{\underline{M}}\right)$ $(\underline{a}=0,1, \cdots, D-1)$ and $E \underline{\alpha}\left(Z^{\underline{M}}\right)(\underline{\alpha}=1, \cdots 2 n)^{3}$, and consider the embedding of $\mathcal{M}_{s w}$ into $M_{T S}$, then for the superembedding to describe a super-p-brane propagating in $M_{T S}$ the pull-back of $E^{a}$ along the Grassmann directions of $\mathcal{M}_{s w}$ must vanish, i.e. in

$$
\left.E^{\underline{a}}\right|_{\mathcal{M}_{s w}}=e^{\underline{a}} E_{a}^{\underline{a}}+e^{\underline{\alpha}} E_{\alpha} \underline{\underline{a}}
$$

the Grassmann components are zero

$$
E_{\alpha} \underline{a}(Z(z))=0
$$

For the most of the superbranes (with some subtleties for the space filling and codimension one branes $[21,22,12,13])$, the superembedding condition (22), accompanied by the $M_{T S}$ and/or $\mathcal{M}_{s w}$ supergravity constraints, implies that

- the geometry of the superworldvolume $\mathcal{M}_{s w}$ is induced by its imbedding into $M_{T S}$, i.e. the $\mathcal{M}_{s w}$ supergravity on the brane is not propagative;

- the dynamics of the superbrane is subject to the standard constraints of the GreenSchwarz formulation, such as the Virasoro constraints and their fermionic counterparts;

\footnotetext{
${ }^{3}$ Note that the supergeometries of $\mathcal{M}_{s w}$ and $M_{T S}$ should be that of corresponding supergravities, which implies that the torsions and curvatures of $\mathcal{M}_{s w}$ and $M_{T S}$ are subject to appropriate supergravity constraints.
} 
- $\kappa$-symmetry is a particular form of worldvolume superdiffeomorphisms;

- the consistency of the superembedding condition results in the same 'brane scan' as that of the GS formulation.

In addition, when the number of the Grassmann directions of $\mathcal{M}_{s w}$ is 16 or higher, the integrability of the superembedding condition requires the worldvolume superfields to satisfy the dynamical equations of motion of the superbrane [23] ${ }^{4}$. It is in this way the covariant equations of motion of the M5-brane were obtained for the first time [7].

\section{SUPEREMBEDDING ACTIONS}

In the cases when the superembedding condition does not imply dynamical equations but only the kinematic constraints, one can construct (doubly supersymmetric) worldvolume superfield actions for corresponding superbranes. Several related methods have been proposed so far to construct the superembedding actions [2, 24, 25, 26, 27, 11, 12, 13].

For the massless superparticles the action is simply the integral over the superworldline of the product of the left hand side of the superembedding condition (22) with a Lagrange multiplier $P_{\underline{a}}^{\alpha}(z)$

$$
S=\int d \xi d^{n} \eta P_{\underline{a}}^{\alpha} E_{\alpha}^{\underline{a}}(Z(z)) .
$$

In the case of the massive superparticles and superbranes to the action (23) one must add a second term which governs the dynamics of the superbrane and produces both the Nambu-Goto (or Dirac-Born-Infeld) term and the Wess-Zumino term of the GS formulation.

Let me explain the general structure of this second term with the example of a space filling D3-brane in $N=2, D=4$ superspace. In this case, the geometry of the worldvolume $\mathcal{M}_{s w}$ is that of chiral $N=1, D=4$ supergravity $[12,13]$.

The D3-brane couples to supergravity fields via the worldvolume pull-back of the Wess-Zumino four-form

$$
\hat{C}=C_{4}+F_{2} \wedge C_{2}+\frac{1}{2} F_{2} \wedge F_{2} C_{0},
$$

where $F_{2}$ is the field strength of the BI gauge field propagating on the D3-brane and $C_{p}$ $(\mathrm{p}=0,2,4)$ are 'Ramond-Ramond' $\mathrm{p}$-form fields.

To construct the D3-brane action as an integral over the chiral $N=1, D=4$ superspace $\mathcal{M}_{s w}$ parametrized by $z_{L}^{M}=\left(\xi_{L}^{m}, \eta^{\alpha}\right)(\alpha=1,2)$ we take a component of the pull-back of (24) onto $\mathcal{M}_{s w}$ which has the appropriate dimensionality. This is $\hat{C}_{\dot{\alpha} \dot{\beta} a b}$. We then contract $\hat{C}_{\dot{\alpha} \dot{\beta} a b}$ with the antisymmetric product of the Pauli matrices $\left(\sigma^{a b}\right)^{\dot{\alpha} \dot{\beta}}$. The

\footnotetext{
${ }^{4}$ This is similar to the case of, say, higher dimensional super-Yang-Mills and supergravity theories whose superfield constraints produce the dynamical equations of motion.
} 
D3-brane action (accompanied by the superembedding term (23)) is

$$
S=\int d^{4} \xi_{L} d^{2} \eta \mathcal{E}_{L}\left(\sigma^{a b}\right)^{\dot{\alpha} \dot{\beta}} \hat{C}_{\dot{\alpha} \dot{\beta} a b}+\text { h.c. },
$$

where $\mathcal{E}_{L}$ is a chiral superspace integration measure (see [12]).

Upon solving for the superembedding condition, integrating over $\eta$ and eliminating auxiliary fields one can get from this simply-looking superembedding action the D3brane action of the GS formulation. It is still a problem for future study to demonstrate how, in the static gauge, the action (25) is related to the super field DBI action discussed in $[29,14,15]$.

As a final remark to this section we note that when the superembedding condition (22) produces dynamical equations of motion and the worldvolume superfield actions cannot be constructed there exist geometrically well-grounded recipes $[28,27]$ of how to construct Green-Schwarz-type actions for corresponding superbranes.

\section{CONCLUSION}

We have tried to describe in simple terms basic features of the superembedding approach, which has not only allowed one to explain and clarify various classical and quantum properties of superstring and superbrane theory, but has also found applications in the construction and description of new superbrane models, and of field theories with partially broken supersymmetry, as well as for solving practical problems. For instance, recently it has been used for calculating vertex operators in (M2-M5)-brane systems [30].

One may expect the superembedding methods to be also useful for other purposes, such as a unified (S-duality) description of fundamental and solitonic extended objects [31], and, in particular, for a covariant description of $N$ coincident Dp-branes and corresponding supersymmetric non-Abelian Dirac-Born-Infeld theories.

\section{ACKNOWLEDGMENTS}

I am grateful to the Organizers of the XXXVII Karpacz Winter School for their warm hospitality, and would like to thank I. Bars, E. Bergshoeff, M. Cederwall, J. de Azcarraga, E. Ivanov, S. Krivonos, J. Lukierski, A. Pashnev, P. Pasti and M. Tonin for interest to this work and discussions. This work was partially supported by the European Commission RTN Programme HPRN-CT-2000-00131 and by the Grant N 2.51.1/52F5/1795-98 of the Ukrainian Ministry of Science and Technology.

\section{REFERENCES}

1. Sorokin, D., Physics Reports, 329, 1-101 (2000).

2. Sorokin, D., Tkach, V. and Volkov, D., Mod. Phys. Lett. A4, 901 (1989). 
3. Berkovits, N., Nucl. Phys. B358, 169 (1991); Ibid B379, 96 (1992); Ibid B420, 332 (1994); Covariant Quantization of the Superstring, hep-th/0008145; Relating the RNS and Pure Spinor Formalisms for the Superstring, hep-th/0104247.

4. Volkov, D. V. and Zheltukhin, A., Lett. Math. Phys. 17, 141 (1989); Nulc. Phys. B335, 723 (1990). Sorokin, D., Tkach, V., Volkov, D. V. and Zheltukhin, A., Phys. Lett. B216, 302 (1989).

Aoyama, S., Pasti, P. and Tonin, M., Phys. Lett. B283, 213 (1992);

Uvarov, D., Covariant $\kappa$-Symmetry Gauge Fixing and the Classical Relation Between Physical Variables of the NSR String and the Type II GS Superstring, hep-th/0104235.

5. Ferber, A., Nucl. Phys. 132, 55 (1977); Shirafuji, T., Progr. Theor. Phys. 70, 18 (1983);

Bengtsson, A. K. H., Bengtsson, I., Cederwall, M. and Linden, N., Phys. Rev. D36, 1766 (1987);

Eisenberg, Y. and Solomon, S., Nucl. Phys. B309, 709 (1988);

Plyushchay, M. S., Phys. Lett. B240, 133 (1990).

6. Nissimov, E., Pacheva, S. and Solomon, S., Nucl. Phys. B296, 469 (1988);

Bandos, I. A., Sov. J. Nucl. Phys. 51, 906 (1990); JETP. Lett. 52, 205 (1990).

7. Howe, P. S. and Sezgin, E., Phys. Lett. B394, 62 (1997); Howe, P. S., Sezgin, E. and West, P. C., Phys. Lett. B399, 49 (1997).

8. Bandos, I., Lechner, K., Nurmagambetov, A., Pasti, P., Sorokin, D. and Tonin, M., Phys. Lett. B408, 135 (1997).

9. Pasti, P., Sorokin, D. and Tonin, M., Phys. Lett. B398, 41 (1997);

Bandos, I., Lechner, K., Nurmagambetov, A., Pasti, P., Sorokin, D. and Tonin, M., Phys. Rev. Lett. 78, 4332 (1997);

Aganagic, M., Park, J., Popescu, C. and Schwarz, J. H., Nucl. Phys. B496, 191 (1997).

10. Hughes, J., Liu, J. and Polchinski, J., Phys. Lett. B180, 370 (1986); Hughes, J. and Polchinski, J., Nucl. Phys. B278, 147 (1986);

Achucarro, A., Gauntlett, J., Itoh, K., and Townsend, P. K., Nucl. Phys. B314, 129 (1989).

11. Pasti, P., Sorokin, D. and Tonin, M., Nucl. Phys. B591, 109 (2000); Geometrical aspects of superbrane dynamics, hep-th/0011020.

12. Bandos, I., Pasti, P., Pokotilov, A., Sorokin, D. and Tonin, M., The space filling Dirichlet 3-brane in $\mathrm{N}=2, \mathrm{~d}=4$ superspace, hep-th/0103152.

13. Drummond, J. M. and Howe, P. S., Codimension zero superembeddings, hep-th/0103191.

14. Bagger, J. and Galperin, A., Phys. Rev. D55, 1091 (1997).

Roc̃ek, M. and Tseytlin, A., Phys. Rev. D59, 106001 (1999).

Ivanov, E., and Krivonos, S., Phys. Lett. B453, 237 (1999).

Kapustnikov, A. and Shcherbakov, A., Linear and nonlinear realizations of superbranes, hepth/0104196.

15. Bellucci, S., Ivanov, E. and Krivonos, S., Phys. Lett. B482, 233 (2000). Superbranes and Super Born-Infeld Theories from Nonlinear Realizations, hep-th/0103136.

16. Brink, L., Deser, S., Zumino, B., Di Vecchia, P. and Howe, P., Phys. Lett. B64, 435 (1976);

Gershun, V. D. and Tkach, V. I., JETP Letters 29, 320 (1979);

Howe, P. S., Penati, P., Pernici, M. and Townsend, P., Phys. Lett. B215, 555 (1988).

17. Neveu, A. and Schwarz, J. H., Nucl. Phys. B31, 86 (1971); Ramond, P., Phys. Rev. D3, 2415 (1971).

18. Howe, P. S. and Tucker, R. W., J. Phys. A10, L155 (1977).

19. De Azcarraga, J. and Lukierski, J., Phys. Lett. B113, 170 (1982);

Siegel, W., Phys. Lett. B128, 397 (1983).

20. Gates Jr., S. J. and Nishino, H., Class. Quantum Grav. 3, 391 (1986).

Kowalski-Glikman, J., van Holten, J. W., Aoyama, S. and Lukierski, J. Phys. Lett. B201, 487 (1987). Kavalov A., and Mkrtchyan, R. L., Spinning superparticles. Preprint Yer.PhI 1068(31)-88, Yerevan, 1988 (unpublished).

21. Howe, P. S., Kaya, A., Sezgin, E. and Sundell, P., Nucl. Phys. B587, 481 (2000).

22. Akulov, V., Bandos, I., Kummer W., and Zima, V., Nucl. Phys. B527, 61 (1998).

23. Galperin, A. and Sokatchev, E., Phys. Rev. D48, 4810 (1993);

Bandos, I., Pasti, P., Sorokin, D., Tonin, M. and Volkov, D., Nucl. Phys. B446, 79 (1995);

Howe, P. S. and Sezgin, E., Phys. Lett. B390, 133 (1997).

24. Tonin, M., Phys. Lett. B266, 312 (1991); Int. J. Mod. Phys. A7, 6013 (1992).

25. Ivanov, E. A. and Kapustnikov, A. A. Phys. Lett. B267, 175 (1991);

Delduc, F. and Sokatchev, E., Class. Quantum Grav. 9, 361 (1992); 
Pashnev, A. and Sorokin, D., Class. Quant. Grav. 10, 625 (1993);

Pashnev, A. and Chikalov, V., Mod. Phys. Lett. A8, 285 (1993); Phys. Rev. D50, 7450 (1994).

26. Galperin, A. and Sokatchev, E., Phys. Rev. D46, 714 (1992); Delduc, F., Galperin, A., Howe, P. S., and Sokatchev, E., Phys. Rev. D47, 587 (1992).

27. Howe, P. S., Raetzel, O. and Sezgin, E., JHEP 9808, 011 (1998).

28. Bandos, D., Sorokin, D. and Volkov, D., Phys. Lett. B352, 269 (1995).

29. Cecotti, S. and Ferrara, S., Phys. Lett. B187, 335 (1987).

30. Moore, G., Peradze, G. and Saulina, N., Instabilities in heterotic M-theory induced by open membrane instantons, hep-th/0012104.

31. Bandos, I., Nucl. Phys. B599, 197 (2001). 